\title{
Patrones epidemiológicos y presentación clínica de los tumores del cuerpo carotídeo
}

\section{Epidemiologic patterns and clinical manifestations of carotid body tumors}

\author{
S. Tatiana Delgado-Aguilar * ${ }^{*}$, José de J. García-Pérez² y Benjamín Sánchez-Martínez ${ }^{3}$ \\ ${ }^{1}$ Servicio de Cirugía Vascular, Victoria Medical Center, Morelia, Michoacán; ${ }^{2}$ Servicio de Cirugía Vascular, Centro Médico Nacional Siglo XXI, Instituto
} Mexicano del Seguro Social (IMSS), Ciudad de México; ${ }^{3}$ Servicio de Ciurgía Vascular; Hospital Ángeles Metropolitano, Ciudad de México. México

\begin{abstract}
Resumen
Objetivos: Los tumores del cuerpo carotídeo (TCC) son neoplasias poco frecuentes, localizadas en la bifurcación carotídea y cuyo diagnóstico generalmente se realiza cuando alcanzan dimensiones importantes. La mayoría son benignos, pero pueden invadir por completo las carótidas y pares craneales. Su crecimiento invasivo llega a ser intracraneal y en algunos casos son irresecables. Por tal motivo, determinar las características epidemiológicas y la asociación de comorbilidades como posibles factores de riesgo para desarrollar dicha patología es de importancia para un diagnóstico oportuno. Material y métodos: Se realizó un estudio retrospectivo y descriptivo, recabando la información contenida en la base de datos del Servicio de Angiología y Cirugía Vascular de todos los pacientes que se sometieron a cirugía de resección de TCC durante el periodo comprendido de enero del 2010 a diciembre del 2014 en el Hospital de Especialidades del Centro Médico Nacional Siglo XXI. Resultados: Se analizaron 91 pacientes, 82 (90\%) pertenecían al sexo femenino y 9 (10\%) al masculino, con una media de edad de 55 años. Los factores de riesgo más importantes fueron el sexo femenino y el sitio de residencia localizado a más de 2,000 metros sobre el nivel del mar (85 pacientes; 93\%), ningún paciente con TCC tenía antecedente de enfermedad pulmonar obstructiva crónica. Conclusiones: Nuestro estudio confirma que el sexo femenino y la altitud son factores de riesgo en nuestra población. La fisiopatología de esta entidad sigue sin comprenderse totalmente; queda descartar algún factor hormonal por la predominancia en mujeres o la posible contribución de factores genéticos.
\end{abstract}

Palabras clave: Tumor del cuerpo carotídeo. Epidemiología. Resultados clínicos.

\section{Abstract}

Background: Carotid body tumors (CBT) are rare neoplasms, located in the carotid bifurcation. The diagnosis is often made by the patient when the tumor reaches important dimensions, they are typically benign, but they can invade carotid vessels and cranial nerves. Growth can extend intracranial hence becoming unrespectable; therefore, the identification of risk factors is crucial for prompt diagnosis. Material and methods: This is a retrospective, descriptive study; medical files from de Vascular Surgery Service at the National Medical Center Siglo XXI in Mexico City were reviewed from patients with CBT who underwent surgery from January 2010 to December 2014. Descriptive analysis was completed. Results: We analyzed 91 patients, 82 (90\%) women, 9 men with a mean age 55 years. The most important risk factors were female gender and home residency at 2000 meters above sea level in 85 (93\%) patients, no patient had history of Chronic Obstructive Pulmonary

Correspondencia:

*S. Tatiana Delgado-Aguilar

E-mail: drasuedelgado@gmail.com

DOI: 10.24875/RMA.M20000012
Disponible en internet: 21-07-2020 Rev Mex Angiol. 2020;48(2):41-46 www.RMAngiologia.com article under the CC BY-NC-ND license (http://creativecommons.org/licenses/by-nc-nd/4.0/). 
Disease. Conclusion: Our study confirms that female gender and geographic altitude are risk factors in our population. The pathophysiology of this entity remains not fully understood, it is important to rule out hormonal factors given the predominance in females and the possible contribution of genetic factors.

Key words: Carotid body tumor. Epidemiology. Clinical outcomes.

\section{Introducción}

El tumor del cuerpo carotídeo es el paraganglioma más frecuente de cabeza y cuello. Es una neoplasia altamente vascularizada, que actúa como quimiorreceptor, localizada en la bifurcación carotídea, con sensibilidad a ciertos estímulos como hipoxia, hipercapnia y acidosis, produce hiperventilación y disminución de la frecuencia cardíaca ${ }^{1,2}$. En general, en las mujeres se presenta con mayor frecuencia que en los hombres, en una relación de 1.9:1. El 57\% de los tumores son del lado derecho, el $25 \%$, del lado izquierdo y el $17 \%$, bilaterales. Aproximadamente el $20 \%$ de los tumores son familiares y tienen un patrón dominante, sin embargo, es más común el tumor del cuerpo carotídeo esporádico que el hereditario en 10 a $50 \%$ de los casos $^{3,4}$. Tienen una malignidad muy baja, menor al $5 \%$, y cerca del $40 \%$ están asociados a mutaciones de los genes $S D H B^{5}$. Estos tumores son asintomáticos en su mayoría, sin embargo, cuando su crecimiento es importante pueden causar disfagia, odinofagia, cefalea, ronquera, dolor de cuello o déficit de pares craneales, por compresión extrínseca y asociado a hipertensión secundaria ${ }^{5}$. Clínicamente se percibe como una tumoración en el triángulo carotídeo, la cual puede acompañarse o no de soplo o thrill. Según el tamaño, extensión e invasión de las estructuras vecinas, la extirpación del tumor puede derivar en serias complicaciones, como lo propuso Shamblin en su clasificación:

- Tipo I: sin compromiso de las estructuras vecinas y fácilmente resecable.

- Tipo II: el más frecuente, con compromiso adherencial a la adventicia, crecimiento circunferencial en la bifurcación carotídea (Figs. 1 A y B)

- Tipo III: marcado compromiso envolvente de la bifurcación carotídea y de los pares craneales, desplazamiento de estructuras vecinas, incluso provocando dolicidad carotídea, como se observa en la figura 2. La lesión de los pares craneales durante la resección representa una complicación frecuente. Hoy en día, el factor que más influye en esta complicación es la extensión del tumor (Figs. 3 A y B) B $^{6,7}$. En esta etapa se ha demostrado que la embolización previa reduce el tamaño del tumor y el sangrado transoperatorio, lo que, aunado con la colocación de un stent en la

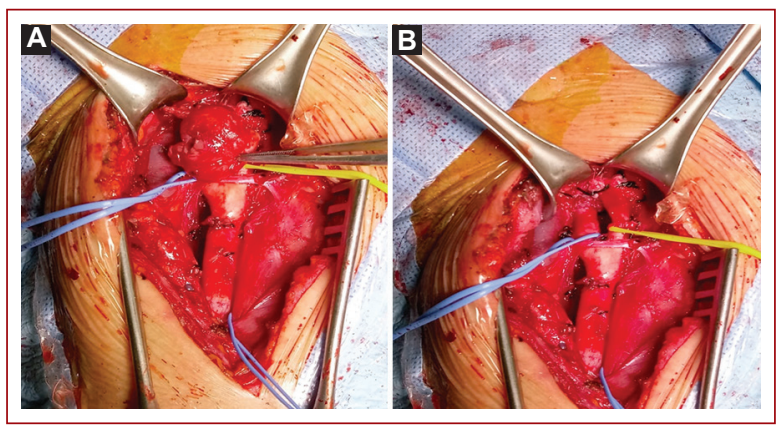

Figura 1. A: se observa un tumor del cuerpo carotídeo Shamblin 2, disecado en la bifurcación carotídea. B: se observa bifurcación carotídea libre de tumor.

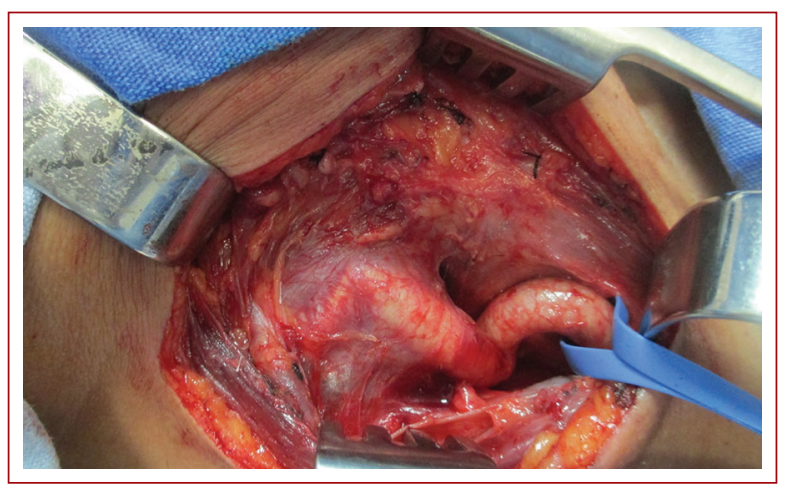

Figura 2. Tumor del cuerpo carotídeo con dolicidad importante de la arteria carótida común.

arteria carótida interna, da como resultado una mejor disección para preservar dicha arteria antes de utilizar injerto vascular ${ }^{8}$.

La mayoría de los pacientes buscan atención médica cuando el tumor pasa del Shamblin I, lo que aumenta la morbilidad $^{9}$. La resección completa es la terapia curativa del tumor y el estándar de oro, en ciertos centros médicos con disección retrocarotídea, que se asocia a menor tiempo quirúrgico y estancia hospitalaria ${ }^{10}$. La clasificación de Shamblin es una herramienta para predecir posibles complicaciones, sangrado y tiempo quirúrgico ${ }^{11}$. Además, tiene un impacto importante en la calidad de vida del paciente, sobre todo a mayor crecimiento $^{12}$. El pronóstico del paciente, además de la etapa en la que se diagnostica, también depende del factor genético, por lo que pacientes con mutaciones 


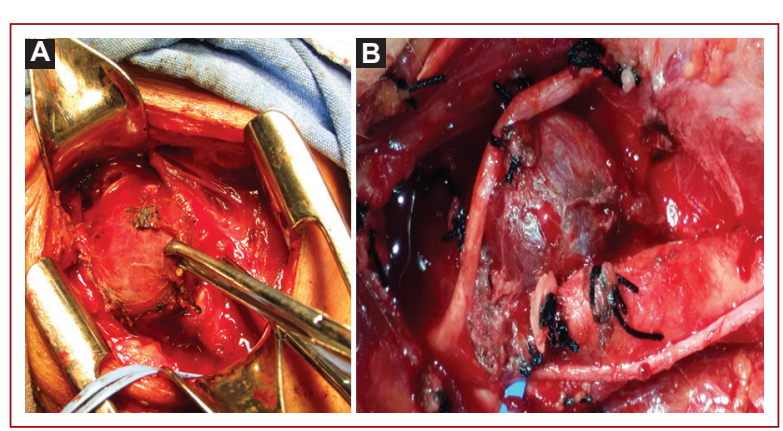

Figura 3. A: tumor del cuerpo carotídeo con gran tamaño e importante involucro de carótidas. B: tumor del cuerpo carotídeo que involucraba carótidas, ya con disección del nervio vago e hipogloso.

de $S D H$ pueden representar un grupo más joven en edad, y con mayor riesgo a malignidad ${ }^{13}$.

El objetivo de este estudio es describir la epidemiología, los patrones demográficos y los factores de riesgo del paciente que pueden presentarse como modificables o no, así mismo, alcanzar un diagnóstico más oportuno y lograr el tratamiento temprano en tumores de diámetro pequeño, lo que disminuye las comorbilidades perioperatorias.

\section{Material y métodos}

Se trata de un estudio retrospectivo, observacional y descriptivo. Se tomó como muestra a los pacientes derechohabientes del Instituto Mexicano del Seguro Social (IMSS) con diagnóstico de tumor del cuerpo carotídeo, referidos de otras unidades, o aquellos espontáneos, a quienes se les dio tratamiento quirúrgico por parte del Servicio de Angiología y Cirugía Vascular en el Hospital de Especialidades del Centro Médico Nacional Siglo XXI en el periodo comprendido del 1 de enero de 2010 al 19 de diciembre de 2014. Se tomaron los datos del archivo clínico del Hospital de Especialidades, así como el registro electrónico de cirugías realizadas del Servicio de Angiología y Cirugía Vascular del Centro Médico Nacional Siglo XXI. El tratamiento quirúrgico del paciente debía corresponder a la fecha establecida, del 1 de enero de 2010 al 19 de diciembre de 2014. De forma inicial, se llevó a cabo una revisión de todos los procedimientos realizados por el Servicio de Angiología y Cirugía Vascular realizados durante el periodo comprendido del 1 de enero de 2010 al 19 de diciembre de 2014. Se recabaron los datos de aquellos pacientes cuyo diagnóstico preoperatorio corresponda a tumor del cuerpo carotídeo. Posterior a la recolección de datos generales del paciente, se solicitó los expedientes clínicos correspondientes al Departamento de Archivo General del Centro Médico Nacional Siglo XXI del IMSS, para complementar la información necesaria. Se incluyeron todos los pacientes que fueron tratados bajo el diagnóstico de tumor del cuerpo carotídeo durante el periodo comprendido de enero de 2010 a diciembre de 2014 y que contaron con el expediente clínico completo disponible para revisión. Las variables y características epidemiológicas incluían la edad, sexo, lado afectado, lugar de residencia y factores de riesgo cardiovasculares. El análisis fue de tipo descriptivo, y se obtuvo media y desviación estándar; en tanto, las variables cualitativas se expresaron de manera nominal y en porcentajes, con representación de los resultados en forma de tablas y gráficas. En el presente proyecto se respetaron las disposiciones institucionales en materia de investigación, y por ser de carácter retrospectivo no requiere carta de consentimiento informado adicional al firmado por el paciente al momento de su ingreso a esta unidad.

\section{Resultados}

En total se encontraron registrados 91 pacientes que se incluyeron en el presente estudio, $82(90 \%)$ mujeres y $9(10 \%)$ hombres. Se sometieron a procedimiento quirúrgico de resección de tumor del cuerpo carotídeo. En cuanto a la edad, los pacientes tenían una edad media de 55 años, una mediana de 59 años y una moda de 57 años, y el rango de edad fue de 19 a 82 años. De todos los pacientes intervenidos, se encontró prevalencia de tumores del cuerpo carotídeo del lado derecho: el $54.95 \%$ fueron del lado derecho, el $38.46 \%$, del lado izquierdo y el $6.59 \%$, bilaterales. De los bilaterales, el $100 \%$ fueron pacientes del sexo femenino. Dentro de los factores de riesgo que se incluyeron en este estudio, los factores de riesgo cardiovasculares tuvieron un papel importante, y se encontraron $35 \mathrm{pa}$ cientes (38.36\%) con hipertensión arterial sistémica, 20 (21.98\%) con diabetes mellitus, 19 (20.88\%) con dislipidemia, $25(27.47 \%)$ con tabaquismo y $0(0 \%)$ con enfermedad pulmonar obstructiva crónica (EPOC) (Fig. 4). Sin duda, el factor de riesgo más importante en el estudio fue el lugar de residencia, siendo casi la totalidad de pacientes residentes del Distrito Federal. De los 91 pacientes incluidos, 85 (93.41\%) eran residentes del Distrito Federal, 2 (2.20\%), de Cuernavaca, $1(1.10 \%)$, de Acapulco, 1 (1.10\%), de Oaxaca, $1(1.10 \%)$, de Querétaro y 1 (1.10\%), de Tuxtla Gutiérrez (Fig. 5). 


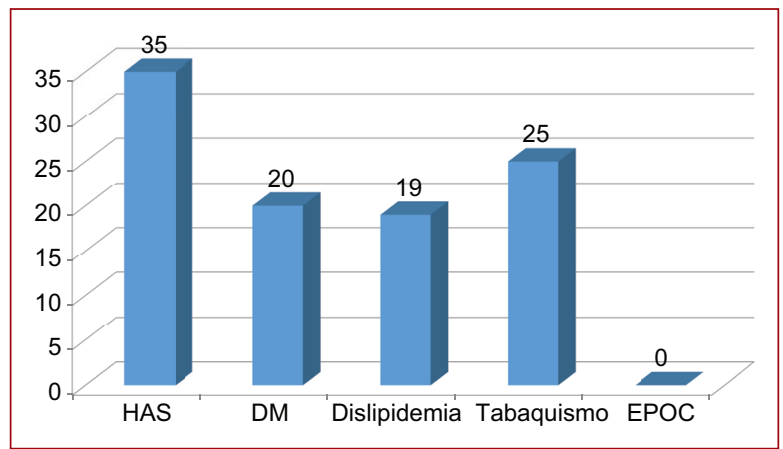

Figura 4. Gráfica de las comorbilidades de los pacientes incluidos en el presente estudio. DM: diabetes mellitus; EPOC: enfermedad pulmonar obstructiva crónica; HAS: hipertensión arterial sistémica.

\section{Discusión}

La epidemiología y la incidencia real del tumor del cuerpo carotídeo en la población general no son específicas, ya que se trata de una patología poco común. Se estima en 1 de cada 30,000 personas. La mayor parte de los tumores se presenta en pacientes que viven en altitudes elevadas. Los tumores del cuerpo carotídeo pueden presentarse como parte de otros síndromes, incluyendo la triada de Carney (sarcoma gástrico estromal, condroma pulmonar, paraganglioma), Von Hippel-Lindau, neurofibromatosis tipo I y neoplasia endocrina múltiple tipo 2. Se han identificado mutaciones específicas, las cuales tienen asociación con paragangliomas. La succinato deshidrogenasa es una enzima mitocondrial compleja que tiene un rol en la fosforilación oxidativa y en la detección de oxígeno intracelular. Las mutaciones de las subunidades $S D H B$ en los cromosomas 1p35-36 y de SDHD en el cromosoma 11q23 resultan en un aumento para presentar paragangliomas y feocromocitoma. Pacientes con mutaciones en SDHD tienen mayor prevalencia de paragangliomas, mientas que los pacientes con mutaciones en $S D H B$ tienen un alto riesgo de malignidad. Existen factores que se han propuesto como predisponentes que aumentan la incidencia de presentar un tumor del cuerpo carotídeo, y el principal es la altitud a más de 2,000 metros, como en Colorado, Perú, México. Otro factor de riesgo que se ha propuesto es la EPOC, ambos factores asociados a hipoxia crónica. El cuerpo carotídeo se desarrolla del tercer arco branquial, el cual contiene células quimiorreceptoras, y detecta cambios en los gases de la circulación arterial, $\mathrm{pH}$ y temperatura. Estimula cambios apropiados en la frecuencia respiratoria, frecuencia cardíaca, tono vasoconstrictor y actividad cortical cerebral.
Ya que el tumor se encuentra fijo a la carótida, es posible movilizar la tumoración en sentido horizontal, pero no verticalmente (signo de Fontaine), así como la reducción de volumen de la tumoración a la compresión, y recuperar su forma original en segundos (signo de Chevassu). El tratamiento de elección es la cirugía, cuyas primeras descripciones datan del siglo XVIII. Fue Scudder, en 1903, quien realizó la primera resección exitosa preservando el sistema carotídeo, y el Dr. Albert quien realizó la primera resección de tumor del cuerpo carotídeo sin dejar secuela neurológica. Von Haller fue el primero en describir en cuerpo carotídeo en 1743. En 1940, Gordon Taylor describió la disección subadventicial, la cual es ampliamente aceptada en la actualidad. Los tumores del cuerpo carotídeo son usualmente detectados en el examen físico, y el diagnóstico se confirma por imagen. El ultrasonido Doppler se considera el estudio de inicio ideal, ya que es no invasivo, y se observa como una masa sólida, bien definida, hipoecoica e hipervascular, ubicada en la bifurcación carotídea, también muestra el grado de infiltración arterial. La angiotomografía nos sirve para delimitar las relaciones del tumor con las estructuras adyacentes y evaluar su extensión craneal. Se observa como una lesión homogénea, de densidad de partes blandas, con notable realce de la densidad por la hipervascularización intrínseca del tumor tras la administración de medio de contraste. La arteriografía se considera como el estándar de oro para el diagnóstico del tumor del cuerpo carotídeo, lo que permite una planificación quirúrgica adecuada y, en algunos casos, realizar embolización preoperatoria de la lesión, con lo que se logra una reducción de su tamaño y disminución del sangrado intraoperatorio. La embolización solo se lleva a cabo inmediatamente posterior a la cirugía. Sin embargo, hay que recordar que la embolización no es un procedimiento inocuo, ya que trae complicaciones como embolismo cerebral, ruptura del tumor, mayor inflamación del tumor, disección carotídea, hemorragia. La angiorresonancia es similar a la arteriografía, pero sin posibilidades de realizar procedimientos endovasculares. El diagnóstico diferencial imagenológico debe realizarse con tumores neurogénicos dependientes del vago o sistema parasimpático, tumores de glándulas salivales, adenopatías metastásicas y más infrecuente hemangiopericitoma parafaríngeo. El tratamiento de elección es la resección quirúrgica de la lesión, la cual es difícil por la invasión de los pares craneales, distorsión de la anatomía, la alta hipervascularidad del tumor y la adherencia a las carótidas. El propósito de este estudio fue identificar 


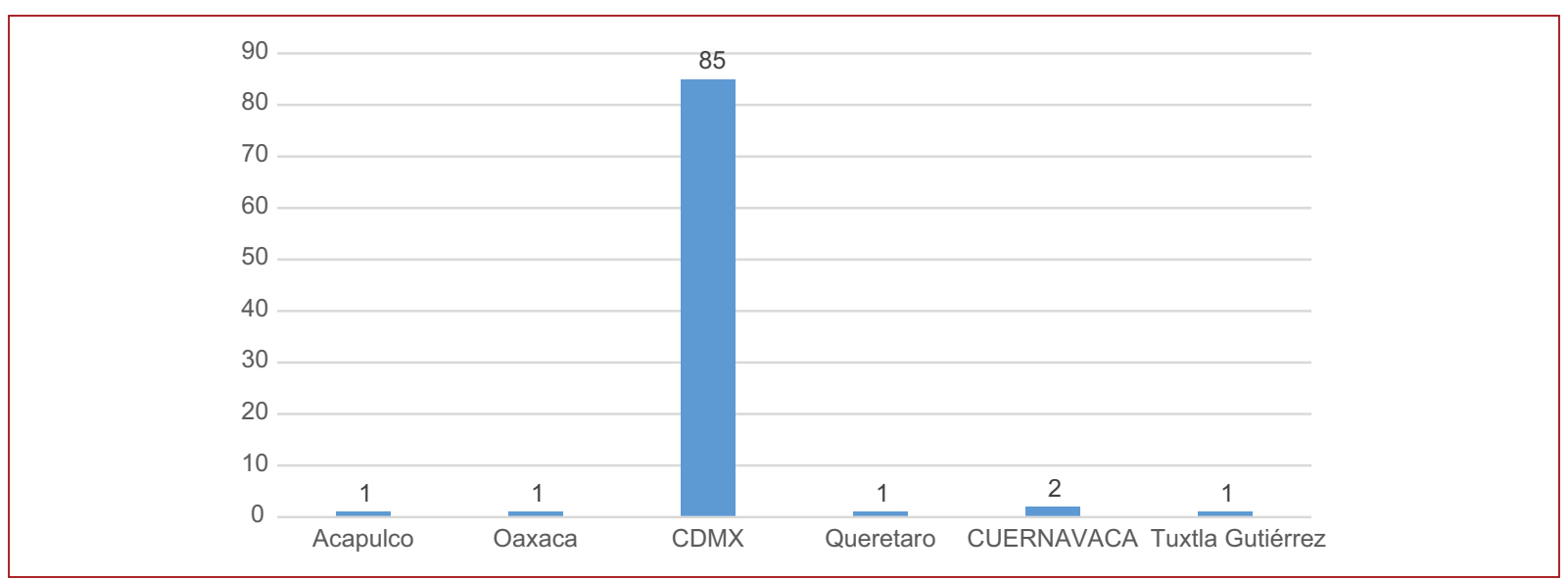

Figura 5. Gráfica de la distribución geográfica del origen de residencia de los pacientes estudiados: un total de 85 $(93 \%)$ radicaba en la zona metropolitana de la Ciudad de México (CDMX).

algún factor de riesgo que fuese estadísticamente significativo para la patología de tumor del cuerpo carotídeo. En segundo lugar, ya identificado el factor de riesgo, catalogarlo como modificable o no, y de esta manera llegar a un diagnóstico más temprano y disminuir la morbimortalidad de este padecimiento, ya que en la mayoría de los pacientes se realiza el diagnóstico cuando el tumor es suficientemente grande para ser percibido por el paciente y siempre se demora porque, la mayor parte de las veces, la patología es subdiagnosticada por ser confundida con adenopatía cervical, por lo que los pacientes han visitado a varios médicos antes de llegar con un angiólogo y cirujano vascular. Se encontró como factores de riesgo importantes la hipertensión arterial sistémica, el género y el lugar de residencia. Este último fue el de mayor peso en el estudio, por lo que la altura se considera como parte esencial de la etiología, ya que el $93.4 \%$ fueron pacientes residentes en el Distrito Federal. Sin embargo, se observaron pacientes residentes de zonas con pobre altura geográfica, lo cual indica que existe parte de la etiología aún sin conocer. Esto coincide con series reportadas previamente en la literatura, las cuales son siempre del Distrito Federal y de Perú por la considerable altura. De manera curiosa se observó que ningún paciente de los incluidos en el estudio presentó EPOC, diagnóstico clínico que no se observa como antecedente ni como diagnóstico en las valoraciones preoperatorias de los pacientes, a pesar de que la literatura lo mencione como factor de riesgo para un tumor del cuerpo carotídeo. La EPOC resultó negativa en el $100 \%$ de los casos, lo cual resulta razonable, ya que la mayoría de los pacientes fueron mujeres con una edad promedio de 55 años.
Se observó un aumento de la incidencia año con año, lo cual se puede deber a un incremento del diagnóstico oportuno o un incremento en la patología misma, la cual pudiera estar asociada a cuestiones ambientales. Sin embargo, no se puede llegar a ninguna conclusión de manera objetiva a partir de estos datos recolectados.

\section{Conclusiones}

La altitud geográfica resultó ser el factor de riesgo más importante para el desarrollo de un tumor del cuerpo carotídeo, por lo que se debe poner particular atención en pacientes residentes en ciudades con altura geográfica importante que acuden por tumoraciones cervicales a revisión médica, así como a pacientes de género femenino, ya que en la mayoría de los pacientes incluidos no se sospechó de tumor del cuerpo carotídeo, sino de adenopatías cervicales, lo que hace que con el tiempo se incremente el tamaño y, con ello, el riesgo perioperatorio. En este estudio, la EPOC no se encuentra como un factor de riesgo para el desarrollo de un tumor del cuerpo carotídeo. Esto puede ser origen de un nuevo estudio, ya que se podría corroborar de manera más objetiva con mediciones de espirometría. El tumor del cuerpo carotídeo sigue siendo una patología poco comprendida, porque aún hoy en día su etiología no se encuentra clara, existen varias teorías, pero definitivamente con esta investigación retrospectiva no se observó la presencia de EPOC en la etiología del mismo. También el estudio deja abierto el campo para una etiología genética y el estudio de factores hormonales, ya que el $90 \%$ de los pacientes fueron mujeres jóvenes. Además, un punto importante a resaltar es que todos los tumores carotídeos bilaterales que se registraron fueron en el sexo femenino, por lo que 
estos dos factores deben orientar a estudios posteriores a esta búsqueda intencional.

\section{Conflicto de intereses}

Los autores no tienen ningún conflicto de intereses.

\section{Responsabilidades éticas}

Protección de personas y animales. Los autores declaran que para esta investigación no se han realizado experimentos en seres humanos ni en animales.

Confidencialidad de los datos. Los autores declaran que han seguido los protocolos de su centro de trabajo sobre la publicación de datos de pacientes.

Derecho a la privacidad y consentimiento informado. Los autores han obtenido el consentimiento informado de los pacientes y/o sujetos referidos en el artículo. Este documento obra en poder del autor de correspondencia.

\section{Bibliografía}

1. Gutiérrez Carreño AR, Sánchez-Fabela $C$, Gutiérrez-Carreño AB, Portillo-Fernández P, Lizola-Margolis RI, Mendieta-Hernández M. Paraganglioma carotídeo. Actualidades 2012. Experiencia de 35 años. Rev Mex Angiol. 2012:40(1):4-12.
2. Garcia-Alva R, Lopez-Pena G, Ricárdez GR, De los Ríos J Ruben-Castillo C, Pérez-Corrales ES, Elenes-Sánchez E, et al. Utilidad de la volumetría de los tumores del cuerpo carotídeo como predictor de sangrado transoperatorio y tiempo quirúrgico. Rev Mex Angiol. 2019;47(3):1-5.

3. Feijoo Cano C, Carranza Martínez JM, Rivera Rodríguez MI, Vázquez Berges I, Herrando Medrano M, Marco Luque MA. Tumores del cuerpo carotídeo, experiencia en 22 años y protocol de segumiento y despistaje familiar. Angiología. 2012;64(4):155-60.

4. Stefano F, Raffaele $\mathrm{G}$. Resection of carotid body tumors reduces arterial blood pressure. An underestimated neuroendocrine syndrome. Int J Surg. 2014;12:563-7.

5. Fishbein L, Merill S, Fraker DL, Cohen DL, Nathanson KL. Inherit mutations in pheocromocytoma and paraganglioma, why all patients should be offered genetic testing. Ann Surg Oncol. 2013;20:14-44.

6. Neumann HPH, Young WF Jr, Eng C. Pheochromocytoma and Paraganglioma. N Engl J Med. 2019;381:552-65.

7. Sajid MS, Hamilton G, Baker DM. Paragangliomas: Epidemiology, clinical presentation, diagnosis and histology. J Vasc Aurg. 2007;34(2):127.

8. Ong HS, Fan XD, Ji T. Radical resecction of a shamblin type III, carotid body tumour without cerebroneurological déficit: improved technique with preoperative embolization and carotid stenting. Int J Oral Maxillofac Surg. 2014:43:1427-30.

9. Economopoulos KP, Tzani A, Reifsnyder T. Adjunct endovascular interventions in carotid body tumors. J Vasc Surg. 2015;61(4):1081-91.e2.

10. Hinojosa CA, Ortiz-Lopez LJ, Anaya-Ayala JE, Orozco-Sevilla V, Nuñez-Salgado AE. Comparison of retrocarotid and caudocranial dissection techniques for the surgical treatment of carotid body tumors. J Vasc Surg. 2015;62(4):958-64.

11. Law $Y$, Chan $Y C$, Cheng SW. Surgical management of carotid body tumor - Is Shamblin classification sufficient to predict surgical outcome? Vascular. 2017;25(2):184-9.

12. García-Alva $R$, Lozano-Corona $R$, Anaya-Ayala JE, Lizola $R$ Lopez-Rocha S, Cuen-Ojeda C, et al. Assessment of health-related quality of life in patients prior to carotid body tumor resection. Vascular. 2019;27(6):612-6.

13. Davila VJ, Chang JM, Stone WM, Fowl RJ, Bower TC, Hinni ML, et al. Current surgical management of carotid body tumors. J Vasc Surg. 2016;64(6):1703-10 